\title{
Tree-level unitarity constraints on the gravitational couplings of higher-spin massive fields
}

\author{
A. Cucchieri and M. Porrati* \\ Department of Physics, New York University, 4 Washington Place, New York, New York 10003 \\ S. Deser ${ }^{\dagger}$ \\ Insititute for Advanced Study, School of Natural Sciences, Princeton, New Jersey 08540 \\ and Theory Division, CERN, CH-1211 Geneva 23, Switzerland
}

(Received 15 August 1994)

\begin{abstract}
We analyze the high-energy behavior of tree-level graviton Compton amplitudes for particles of mass $m$ and arbitrary spin, concentrating on a combination of forward amplitudes that will be unaffected by eventual cross couplings to other, higher spins. We first show that for any spin $>2$, tree-level unitarity is already violated at energies $\sim \sqrt{m M_{\mathrm{Pl}}}$, rather than at the Planck scale $M_{\mathrm{Pl}}$, even for $m \ll M_{\mathrm{Pl}}$. We then restore unitarity to this amplitude up to $M_{\mathrm{Pl}}$ by adding nonminimal couplings that depend on the curvature and its derivatives, and modify the minimal description, including particle gravitational quadrupole moments at $\sim m^{-1}$ scales.
\end{abstract}

PACS number(s): 11.15.Bt, 04.60.- $\mathrm{m}, 04.62 .+\mathrm{v}, 11.90 .+\mathrm{t}$

\section{INTRODUCTION}

It is fortunate that no higher $(s>2)$ spin elementary excitations have been observed: massless ones are well known to suffer from grave consistency problems when they, unavoidably, couple to gravity ${ }^{1}[1]$; massive ones, with which we will be concerned here, are not directly touched by this difficulty, related to the loss of free-field gauge invariance when ordinary derivatives are replaced by covariant ones. Instead, we shall see that they inherit from their $m=0$ parts an obstacle of a more intuitive kind, namely that tree-level unitarity is already violated at energies $\sqrt{s} \sim \sqrt{m M_{\mathrm{Pl}}}$, well below Planck mass $M_{\mathrm{Pl}}$ for ordinary $m$ in, e.g., graviton Compton scattering. One of the motivations for our study is the fact that massive excitations of any spin are necessarily present in the context of string theory, at least at levels where particle expansion is meaningful. Those masses can be much smaller than the Planck scale if the string is weakly interacting; thus, one would like to see how field theory arranges itself "optimally" in this context and compare this with the description arising from the $\beta$-function expansion, say, of the string.

A very similar problem arises in the electromagnetic coupling of higher-spin charged fields, and provides another motivation for the gravitational analysis. In the

\footnotetext{
${ }^{*}$ Also at Theory Division, CERN, CH-1211 Geneva 23, Switzerland.

${ }^{\dagger}$ Permanent address: Department of Physics, Brandeis Univ., Waltham, MA 02254-9110.

${ }^{1} \mathrm{~A}$ consistent scheme may perhaps be achieved in the presence of a negative cosmological constant $\Lambda$ [2]. Here we deal only with $\Lambda=0$ gravity.
}

Maxwell case, it was found [3] that judicious addition of nonminimal, Pauli, couplings restores low-energy tree unitarity in Compton scattering with the added bonus that the new terms give rise to a universal value $g=2$ of the $g$ factor for all spins. The corresponding question here would be about the gravitational quadrupole moments of the matter system. The first example, $s=\frac{5}{2}$, of our topic was treated recently [4]; both the gravitational Compton amplitude difficulty and its (partial) cure were exhibited. Here we shall generalize these results to cover all bosonic and fermionic spin $\geq \frac{5}{2}$. We shall also emphasize that there are in fact two very different contributions to the graviton-particle vertex or current, defined with respect to the gauge transformations permitted by the massless parts. Here we shall deal with the "gaugelongitudinal" parts of the currents, where our nonminimal completion will indeed improve the high-energy behavior. These new terms will also contribute to (but not remove) the unwanted behavior of the "gauge-transverse" currents; we conjecture that more radical means will be required here, including (at least) the addition of infinite Regge towers of higher-spin modes, and corresponding corrections to the Einstein action, as automatically happens in string theory.

Let us briefly review the problems of and differences between massive and massless spin $>\frac{1}{2}$ fields. The field equations of the latter are always of the schematic form $D_{\mu} F^{\mu \nu \cdots}=0$ for bosons, where $F$ is the antisymmetric field strength. Hence the Bianchi identity $2 D_{\nu} D_{\mu} F^{\mu \nu \cdots}=\left[D_{\nu}, D_{\mu}\right] F^{\mu \nu \cdots}$ will lead to unacceptable local constraints of the form $R F=0$ on the matter field and full curvature tensor $R$ (only spin 1 and 2 escape this difficulty). A similar problem occurs for fermions, where $F$ is proportional to the fermionic potential itself (here only spin $\frac{3}{2}$ escapes because just the Ricci tensor enters in the above constraint). In the massless case, there can be no nonminimal help for these problems, by gauge invari- 
ance and dimensions. For massive models, on the other hand, the loss of Bianchi identities is not as catastrophic because the resulting constraints simply relate the $R F$ term to terms $\sim m^{2} D_{\mu} \phi^{\mu \cdots}$, where $\phi$ is the bosonic amplitude, for example, thereby merely shifting the usual $m^{2} \partial_{\mu} \phi^{\mu \cdots}=0$ condition of flat space. Furthermore, once a mass parameter is present, it becomes possible to add terms such as $\sim m^{-1} R \bar{\psi} \psi$ (for fermions), a mechanism we will indeed exploit here. There are two equivalent ways of performing the analysis: either directly, at the level of the matter field equations (where one can in turn either attempt an all-order analysis or expand in powers of the metric deviation $h_{\mu \nu}=g_{\mu \nu}-\eta_{\mu \nu}$ from flatness), or by considering the Compton scattering amplitude, where the field equations' characteristics are divided into their linear parts in $h_{\mu \nu}$ (the vertices) and their flat-space parts (the propagators). Here we proceed in the latter way, the fundamental difference, for our purposes, between $s \leq 2$ and $s>2$ fields being that, for the former, the scattering amplitudes remain small up to the Planck scale. In order to convince ourselves that this lower-spin property is not obvious, let us consider, for instance, the elastic scattering of a graviton off a massive spin- $s$ particle, depicted in Fig. 1.

The corresponding scattering amplitude involves the propagator $\Pi$ of the massive field $\phi$. For $s \geq 1$ this propagator contains, in its numerator, terms proportional to $1 / \mathrm{m}^{2}$, related to the existence of (restricted) gauge invariances in the $m \rightarrow 0$ limit. These mass singularities could, in principle, give rise to a scattering amplitude containing terms $O\left(s^{2} / m^{2} M_{\mathrm{Pl}}^{2}\right)$. Such a scattering amplitude would become large, and eventually exceed the unitarity bounds, at $\sqrt{s} \approx \sqrt{m M_{\mathrm{Pl}}}$. If $m \ll M_{\mathrm{Pl}}$ this energy lies far below the Planck scale; also, the amplitude would have no massless limit due to its dependence on powers of $m^{-1}$.

The reason why $O\left(s^{2} / m^{2} M_{\mathrm{Pl}}^{2}\right)$ terms are absent for $s=1, \frac{3}{2}, 2$ is the following: The diagrams in Fig. 1 giving rise to the dangerous $O\left(s^{2} / m^{2} M_{\mathrm{Pl}}^{2}\right)$ terms have the form ${ }^{2} J \Pi J$. The tensor current $J$ is obtained by varying the action $S\left[\phi, e_{\mu}^{a}\right]$ with respect to the field $\phi$, and keeping only terms linear in the fluctuation of the metric or vierbein $^{3}$ about the flat-space background. This vertex is thus universally defined by

$$
\left.\frac{\delta S\left[\phi, e_{\mu}^{a}\right]}{\delta \phi}\right|_{L} \equiv J
$$

As noticed above, terms proportional to $1 / \mathrm{m}^{2}$ in the propagator $\Pi$ are related to gauge invariance in the massless limit. More precisely $\Pi \stackrel{\circ}{\phi}=m^{-2} \stackrel{\circ}{\phi}$ iff $\stackrel{\circ}{\phi}$ is a pure gauge. The standard form of $\stackrel{\circ}{\phi}$ for $s=1, \frac{3}{2}, 2$ reads

\footnotetext{
${ }^{2}$ The "seagull" diagram in Fig. 1 does not contribute to the leading zero-mass singularity.

${ }^{3} \mathrm{By}$ working in the symmetric gauge $e_{\alpha \mu}=e_{\mu a}$ for the vierbein, we have $e_{\mu a}=\eta_{\mu a}+\frac{1}{2} h_{\mu a}$.
}
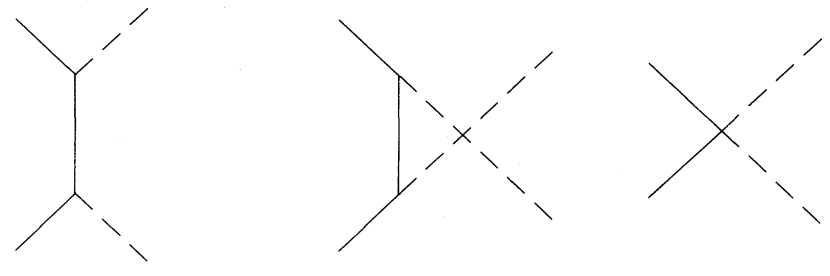

FIG. 1. The tree-level graviton Compton diagrams; solid lines denote massive, spin-s particles, dashed lines denote gravitons.

(throughout, we consider Majorana spinors and real tensors, for simplicity)

$$
\begin{array}{ll}
s=1: & \stackrel{\circ}{\phi}_{\mu}=\partial_{\mu} \epsilon, \quad s=\frac{3}{2}: \quad \stackrel{\circ}{\phi}_{\mu}=\partial_{\mu} \epsilon \\
s=2: & \stackrel{\circ}{\phi}_{\mu \nu}=\partial_{\mu} \epsilon_{\nu}+\partial_{\nu} \epsilon_{\mu} .
\end{array}
$$

The gauge parameter $\epsilon$ is a scalar for $s=1$, a spinor for $s=\frac{3}{2}$, and a vector for $s=2$. If the projection of the current $J$ on the vectors $\stackrel{\circ}{\phi}$, denoted by $J \cdot \stackrel{\circ}{\phi}$, has the form $m X$, with $X$ any operator possessing a smooth $m \rightarrow 0$ limit, then, by dimensions, no $O\left(s^{2} / m^{2} M_{\mathrm{Pl}}^{2}\right)$ terms will arise in the scattering amplitude of Fig. 1 . Here the key observation is that, up to $O\left(h^{2}\right)$ terms, $J \cdot \dot{\phi}$ equals $\stackrel{\circ}{\phi} \cdot \delta S\left[\phi, e_{\mu}^{a}\right] / \delta \phi$, due to Eq. (1.1): we find the projection $J \cdot \stackrel{\circ}{\phi}$ by varying the action $S\left[\phi, e_{\mu}^{a}\right]$ under a gauge transformation, ${ }^{4}$ and linearizing in the gravitational field $h_{\mu \nu}$. For generic spin this variation contains terms of the form $m X$, hereafter called "soft," as well as hard terms. The hard terms do not vanish in the $m \rightarrow 0$ limit. ${ }^{5}$ For $s=\frac{3}{2}$ and 2 , though, the hard terms are proportional to the (linearized) Ricci tensor $R_{\mu \nu}[5,6]$; they are absent altogether for $s=1$. These $s=\frac{3}{2}, 2$ hard contributions thus vanish when we impose the free-graviton equations of motion (that is the linearized Einstein equations in the vacuum) that the external gravitons in Fig. 1 obey.

Having recalled how the lower spins escape the treeunitarity problem, we will turn, in the rest of this paper, to the general case. In Sec. II, we review the first system, $s=\frac{5}{2}$, in which the full extent of the high-energy-limit problem is manifested, and isolate the specific forward scattering amplitude to be analyzed. We will then consider the general integer spin case in Sec. III, and find the required nonminimal additions that remove the hard contributions to that amplitude. The construction is extended to all half-integer spin systems in Sec. IV, while

\footnotetext{
${ }^{4}$ To be exact, under a restricted gauge transformation of the massless free Lagrangian, $S_{0}[\phi]=\lim _{m \rightarrow 0} S[\phi]$.

${ }^{5}$ The nonvanishing of $J \cdot \stackrel{\circ}{\phi}$ in the massless limit implies, by (1.1), that the equations of motion of a massless high-spin particle are generically inconsistent [1].
} 
Sec. V contains some concluding remarks and interesting open problems.

\section{REVIEW OF SPIN $\frac{5}{2}$}

Before going on to the general case, it is instructive to summarize, following [4], the situation for spin $\frac{5}{2}$, where one is first faced with hard terms involving the full curvature. The system is described, as in $[8,9]$, by a symmetric tensor-spinor $\psi_{\mu \nu}$, and an auxiliary spinor $\chi$. The corresponding action was written, as in $[9,7]$,

$$
\begin{aligned}
S= & \int d^{4} x \sqrt{g}\left[-\frac{1}{2} \bar{\psi}_{a b} D \psi_{a b}-\bar{\psi}_{a b} \gamma_{b} D \gamma_{c} \psi_{c a}\right. \\
& +2 \bar{\psi}_{a b} \gamma_{b} D_{c} \psi_{c a}+\frac{1}{4} \bar{\psi}_{a a} D \psi_{b b}-\bar{\psi}_{a a} D_{b} \gamma_{c} \psi_{b c} \\
& +\frac{m}{2}\left(\bar{\psi}_{a b} \psi_{a b}-\frac{3}{4} \bar{\psi}_{a b} \gamma_{b} \gamma_{c} \psi_{c a}-\frac{7}{4} \bar{\psi}_{a a} \psi_{b b}\right. \\
& \left.\left.-\frac{16}{3} \bar{\chi} \psi_{a a}-\frac{32}{9} \bar{\chi} \chi\right)\right]
\end{aligned}
$$

The conventions on the metric and $\gamma$ matrices follow [7] and the covariant derivative of the field $\psi_{\mu \nu}$ is

$$
D_{\mu} \psi_{\nu \rho}=\partial_{\mu} \psi_{\nu \rho}+\frac{1}{2} \sigma_{a b} \omega_{\mu}^{a b}(e) \psi_{\nu \rho}-\Gamma_{\mu \nu}^{\lambda} \psi_{\lambda \rho}-\Gamma_{\mu \rho}^{\lambda} \psi_{\nu \lambda}
$$

The free spin- $\frac{5}{2}$ Lagrangian possesses a restricted gauge invariance at $m=0[9,10]$. The gauge parameter is a $\gamma$-traceless vector spinor, and the gauge transformation reads

$$
\delta \psi_{\mu \nu}=\partial_{\mu} \epsilon_{\nu}+\partial_{\nu} \epsilon_{\mu}, \quad \gamma^{\mu} \epsilon_{\mu}=0
$$

The free equations of motion of $\psi_{\mu \nu}$ and $\chi$ give, as expected

$$
\not \partial \psi_{\mu \nu}=m \psi_{\mu \nu}, \quad \gamma^{\mu} \psi_{\mu \nu}=\partial^{\mu} \psi_{\mu \nu}=0, \quad \chi=0 .
$$

In order to see whether $O\left(s^{2} / m^{2} M_{\mathrm{Pl}}^{2}\right)$ terms are present in the scattering diagrams of Fig. 1 one must perform a variation of the action (2.1) under the transformation (2.3), linearize in the gravitational field, and put $\psi_{\mu \nu}, \chi$, and $h_{\mu \nu}$ on shell. A short calculation gives [4]

$$
\delta S=-\int d^{4} x 2 \sqrt{g} \bar{\epsilon}_{\nu} \gamma_{\rho} \psi_{\lambda \sigma} R^{\nu \lambda \rho \sigma}+\text { soft terms }+O\left(h^{2}\right) .
$$

The hard term in this equation is proportional to the Riemann tensor; thus, it does not vanish on shell, so the scattering amplitude of Fig. 1 does contain $O\left(s^{2} / m^{2} M_{\mathrm{P} 1}^{2}\right)$ terms.

Thus, a minimally coupled light $\left(m \ll M_{\mathrm{Pl}}\right)$ spin- $\frac{5}{2}$ field interacts strongly with gravity even at relatively low energies $\left(\sqrt{s} \approx \sqrt{m M_{\mathrm{Pl}}} \ll M_{\mathrm{Pl}}\right)$, and the presence of in- verse powers of the mass also bars us from taking the massless limit. This scenario is rather bizarre: it would seem more natural that gravitational interactions remain weak up to Planckian energies $\sqrt{s} \approx M_{\mathrm{Pl}}$, irrespective of any particle's mass. In [4] it was shown that in order to implement this requirement one must add, to the minimal Lagrangian (2.1), a nonminimal term proportional to the Riemann curvature whose gauge variation cancels the hard term given in (2.5). In other words, in order to obtain gravitational tree-level amplitudes that may possess a smooth $m \rightarrow 0$ limit one must add a nonminimal term such that the high-spin current in (1.1), i.e., $J_{\mu \nu} \equiv \delta S[\psi] /\left.\delta \psi_{\mu \nu}(x)\right|_{L}$, is conserved up to terms proportional to $m$.

The required nonminimal term turned out to be ${ }^{6}$ [4]

$$
\begin{gathered}
-\frac{1}{2} m^{-1} \int d^{4} x \sqrt{g} \bar{\psi}_{\mu \nu} R^{ \pm \mu \rho \nu \sigma} \psi_{\rho \sigma} \\
R^{ \pm \mu \rho \nu \sigma}=R^{\mu \rho \nu \sigma} \pm \frac{1}{2} \gamma^{5} \epsilon^{\nu \sigma \alpha \beta} R_{\alpha \beta}^{\mu \rho}
\end{gathered}
$$

Actually the improvement (2.6) does not by itself accomplish the desired goal of removing all singular terms from the scattering amplitude. The fermionic current $J_{\mu \nu}$ has two parts: the pure spin $\frac{5}{2}$, transverse and $\gamma$-traceless one, and its longitudinal complement, the (lower spin) part in the direction of the gauge variation (2.3). The nonminimal addition has ensured that the contribution of the latter is well behaved in the massless limit. However (2.6) also contributes a (singular) part to the transverse current, and the latter will contribute already against the nonsingular parts of the propagator $\Pi$. We believe that in order to cancel this mass singularity one would be forced, at least, to add other massive fields, of spin larger than $\frac{5}{2}$. Probably the process never stops until an entire Regge trajectory has been added. A similar conjecture has been made in [11], in the case of purely gravitational interactions of elementary particles, and has been used in [12] as one of the early motivations to consider string theory as the natural setting of a perturbative theory of quantum gravity. In terms of a field equation analysis, the addition (2.6) only ensures that the longitudinal projection of the matter equations obeys Bianchi identities devoid of explicit curvature terms; but it does not remove such (singular) terms in the transverse complement.

The implementation of tree-level unitarity on all scattering amplitudes is a most interesting program, especially because the (conjectured) need for new (infinitely many) degrees of freedom seems to lead naturally to string theory. On the other hand this program seems difficult to achieve concretely at present, and thus we prefer to adopt another strategy, namely, to find scattering amplitudes that do not depend on the transverse

\footnotetext{
${ }^{6}$ At $R_{\mu \nu}=0$ the quantities $R^{ \pm \mu \rho \nu \sigma}$ reduce, on fermionic states of definite chirality, to the self-dual (or anti-self-dual) components of the Weyl tensor $C^{\mu \rho \nu \sigma}$.
} 
current, and implement tree-level unitarity only on those amplitudes. In other words, we prefer to divide the implementation of tree-level unitarity into two parts. One will give rise to constraints on the interactions of individual high-spin particles alone; the other will not only further constrain these interactions but also require the introduction of new degrees of freedom.

Fortunately, an amplitude independent of the transverse part of the current $J$ is known. It can be written for particles of arbitrary spin, and reads

$$
f_{-}\left(E, s_{3}\right)=\frac{1}{2 E}\left[f\left(E, s_{3},+\right)-f\left(E, s_{3},-\right)\right] .
$$

It is the difference between the positive- and negativehelicity forward elastic scattering amplitude of a graviton of energy $E$ off a spin- $s$ particle at rest; $-s \leq s_{3} \leq s$ is the helicity of the spin- $s$ particle. The equivalent amplitude, in the case of photon scattering off a spin- $s$ target, was used in [13] to constrain the form of electromagnetic interactions of high-spin particles, by using appropriate dispersion relations. The natural question is then whether the cancellation of hard terms in (2.7) is specific to spin $\frac{5}{2}$ or whether it holds in general. We shall show how the results of [4] can be extended to massive particles of any spin, provided suitable gravitational quadrupoles are added to the Lagrangian: in this respect, spin $\frac{5}{2}$ is not special.
The fact that the forward elastic scattering amplitude $f_{-}\left(E, s_{3}\right)$ is tree-level unitary means that there exists a way of defining the gravitationally coupled action such that the current $J$, defined in Eq. (1.1) is softly broken; namely, as explained before, that its divergence is proportional to $m$. If one could take the $m \rightarrow 0$ limit of (1.1), one would then obtain an exactly conserved tensor current, which would imply the existence of a large "softly broken" higher-spin symmetry. To justify this conjecture one may appeal to the case of the standard-model $W$ bosons in an external em field: in that case the role of the currents $J$ is played by the off-diagonal components of the $\mathrm{SU}(2)$ gauge group. ${ }^{7}$ The $m \rightarrow 0$ limit may be legitimate in a completely consistent theory of interacting higher spins, as string theory is conjectured to be. The massless limit there may correspond to the tensionlessstring limit $\alpha^{\prime} \rightarrow \infty[15,16]$ or to a new topological phase of gravity [17].

\section{INTEGER SPINS}

To compute the amplitude (2.7) for arbitrary spin we need an explicit form of the free massive, spin- $s$ field Lagrangian; we use that of [8], which is written in terms of symmetric, traceless tensors $\phi^{p}$ of rank $p=s, s-2, s-$ $3, \ldots, 0$ :

$$
\begin{aligned}
L= & \frac{1}{2} \phi^{s}\left(\partial^{2}-m^{2}\right) \phi^{s} \\
& +\frac{s}{2} \phi^{s} \cdot \overleftarrow{\partial} \partial \cdot \phi^{s}+\frac{s(s-1)^{2}}{2 s-1}\left[\phi^{s-2} \partial \cdot \partial \cdot \phi^{s}-\frac{1}{2} \phi^{s-2}\left(\partial^{2}-\frac{s}{s-1} m^{2}\right) \phi^{s-2}+\frac{(s-2)^{2}}{2(2 s-1)} \phi^{s-2} \cdot \overleftarrow{\partial} \partial \cdot \phi^{s-2}+\cdots\right]
\end{aligned}
$$

where $\partial \cdot \phi^{p} \equiv \partial^{\mu} \phi_{\mu \mu_{2} \cdots \mu_{p}}^{p}$. This Lagrangian involves the minimal number of auxiliary (nonpropagating) fields needed in order to describe the propagation of a pure spin- $s$ field. Since we shall consider only perturbative $S$ matrix elements, any other consistent Lagrangian should give rise to equivalent results. The equations of motion obtained from (3.1) are

$$
\phi^{p<s}=0, \quad\left(\partial^{2}-m^{2}\right) \phi^{s}=0, \quad \partial \cdot \phi^{s}=0 .
$$

The Lagrangian (3.1) may now be coupled to gravity using the minimal substitution

$$
\partial_{\mu} \rightarrow D_{\mu}, \quad \eta^{\mu \nu} \rightarrow g^{\mu \nu}
$$

The resulting minimal [with respect to the derivative or- dering in (3.1) action $^{8} S_{m}\left[\phi, g_{\mu \nu}\right]$ gives rise to a current $J$ as in (1.1). As we explained in the Introduction (and illustrated for the case of spin $\frac{5}{2}$ ) the longitudinal part of $J$ is associated with a restricted gauge invariance of

\footnotetext{
${ }^{7}$ The link between tree-level unitarity and gauge symmetry indeed holds for any system of interacting particles of spin $\leq 1[11,14]$.

${ }^{8} \mathrm{Minimal}$ coupling to any gauge theory, including gravity, is of course only defined uniquely for systems of first derivative order (fermions or first-order form of bosons); for bosons of nonzero spin, different order of derivatives will differ by curvature terms. This ambiguity is unimportant; the real question is whether any given ordering, corrected if necessary by nonminimal, "Pauli," terms proportional to the curvature or its derivatives, can lead to the desired amplitude behavior.
} 
the massless free Lagrangian. More precisely, one varies $S_{m}\left[\phi, g_{\mu \nu}\right]$ with respect to the gauge transformation [3]

$$
\begin{aligned}
\delta \phi_{\mu_{1} \cdots \mu_{s}}^{s} & =\partial_{\left(\mu_{1}\right.} \epsilon_{\left.\mu_{2} \cdots \mu_{s}\right)}-\frac{s-1}{2 s} g_{\left(\mu_{1} \mu_{2}\right.} \partial^{\lambda} \epsilon_{\left.\mu_{3} \cdots \mu_{s}\right) \lambda} \\
\delta \phi_{\mu_{1} \cdots \mu_{s-2}}^{s-2} & =\frac{2 s-1}{s^{2}} \partial^{\lambda} \epsilon_{\mu_{1} \cdots \mu_{s-2} \lambda} \\
\delta \phi_{\mu_{1} \cdots \mu_{p}}^{p} & =0 \text { if } p<s-2
\end{aligned}
$$

where the parentheses mean total (normalized) symmetrization and the gauge parameter $\epsilon_{\mu_{1} \cdots \mu_{s-1}}$ is a symmetric, traceless, rank- $(s-1)$ tensor, obeying the equation

$$
\partial^{\mu} \partial^{\lambda} \epsilon_{\mu \lambda \mu_{3} \cdots \mu_{s-1}}=0
$$

The variation of the action under the transformation (3.4) gives, after the free equations of motion for the fields $\phi^{p}$ and $h$ are used,

$$
\begin{aligned}
\delta S_{m}= & \int d^{4} x \sqrt{-g} 2(s-1) \epsilon_{\gamma}{ }^{\mu_{2} \cdots \mu_{s-1}} R^{\alpha \beta \gamma \delta} \partial_{\alpha} \phi_{\beta \mu_{2} \cdots \mu_{s-1} \delta}^{s} \\
& + \text { soft terms }+O\left(h^{2}\right)
\end{aligned}
$$

The hard terms, proportional to the Riemann tensor and to $(s-1)$, vanish for spin $s=1$ but do not vanish on shell. Equation (3.6) is simply the projection of the current $J$ over the gauge directions: it is proportional to the longitudinal part of $J$. Since the propagator $\Pi$ of the massive field $\phi$ is singular as $1 / \mathrm{m}^{2}$ along those gauge directions, to obtain a well-behaved scattering amplitude $f_{-}\left(E, s_{3}\right)$ one must add to the minimal Lagrangian a new term $S_{n m}$ such that $\delta S_{m}+\delta S_{n m}$ is soft. This term is

$$
S_{n m}=\frac{s(s-1)}{2} \int d^{4} x \sqrt{-g} \phi_{\alpha \gamma}^{s}{ }^{\mu_{3} \cdots \mu_{s}} R^{\alpha \beta \gamma \delta} \phi_{\beta \delta \mu_{3} \cdots \mu_{s}}^{s} .
$$

The variation of the new nonminimal action $S=S_{m}+$ $S_{n m}$ gives

$$
\begin{aligned}
\delta S= & -\int d^{4} x \sqrt{-g}(s-1)(s-2) \epsilon_{\alpha \gamma}^{\mu_{3} \cdots \mu_{s-1}} \\
& \times\left(\partial^{\lambda} R^{\alpha \beta \gamma \delta}\right) \phi_{\beta \delta \mu_{3} \cdots \mu_{s-1} \lambda}^{s} \\
& + \text { soft terms }+O\left(h^{2}\right) .
\end{aligned}
$$

Again, one finds that there are hard terms nonvanishing on shell, but now they are zero for $\operatorname{spin} s<3$. It is interesting to notice that this result, in agreement with [18], can be obtained directly from some "minimal" action by exploiting the ambiguity of the minimal coupling, i.e., without introducing the term (3.7). In fact, if we minimally couple the free Lagrangian (3.1) to gravity after integrating the second term by parts, and exchanging the order of the derivatives, namely, if we use the term $(s / 2) \partial_{\beta} \phi^{s \alpha \mu_{2} \cdots \mu_{s}} \partial_{\alpha} \phi_{\mu_{2} \cdots \mu_{s}}^{s \beta}$ instead of $(s / 2) \partial_{\alpha} \phi^{s \alpha \mu_{2} \cdots \mu_{s}} \partial_{\beta} \phi^{s \beta}{ }_{\mu_{2} \cdots \mu_{s}}$, the variation (3.8) is obtained directly. By using the identity

$$
\begin{aligned}
D_{\alpha} D_{\beta} \phi_{\mu_{1} \cdots \mu_{s}}^{s} & \equiv\left(D_{\beta} D_{\alpha}+\left[D_{\alpha}, D_{\beta}\right]\right) \phi_{\mu_{1} \cdots \mu_{s}}^{s} \\
& \equiv D_{\beta} D_{\alpha} \phi_{\mu_{1} \cdots \mu_{s}}^{s}+s R_{\alpha \beta \mu_{1}}{ }^{\tau} \phi_{\tau \mu_{2} \cdots \mu_{s}}^{s}
\end{aligned}
$$

one can verify that the minimal action defined in this way is different from the previous nonminimal one $S_{m}+S_{n m}$ only through terms proportional to the Ricci tensor, that is through terms vanishing on graviton shell. This confirms what was sketched in the Introduction: the minimal coupling gives rise to hard terms in the scattering diagrams of Fig. 1 only for spins $s \geq 3$. Moreover, this new minimal action is equivalent to the first-order formalism [6]; in fact, its variation under the transformation (3.4), with standard derivatives replaced by covariant ones and by using the identity (our convention is $R_{\mu \alpha \beta}^{\tau} \sim+\partial_{\alpha} \Gamma_{\beta \mu}^{\tau}$ and the signature is mostly minus) $D^{\alpha} R_{\alpha \beta \gamma \delta} \equiv D_{\delta} R_{\gamma \beta}-D_{\gamma} R_{\beta \delta}$, gives

$$
\begin{aligned}
\delta S= & \int d^{4} x \sqrt{-g}\left\{-(s-1)(s-2) \epsilon_{\alpha \gamma}^{\mu_{4} \cdots \mu_{s}}\left[\left(D^{\lambda} R^{\alpha \beta \gamma \delta}\right) \phi_{\lambda \beta \delta \mu_{4} \cdots \mu_{s}}^{s}+2 R^{\alpha \beta \gamma \delta} D^{\lambda} \phi_{\lambda \beta \delta \mu_{4} \cdots \mu_{s}}^{s}\right]\right. \\
& \left.+(s-1) \epsilon_{\alpha}^{\mu_{3} \cdots \mu_{s}}\left[-2\left(D^{\lambda} R^{\alpha \beta}\right)+\left(D^{\alpha} R^{\lambda \beta}\right)-2 R^{\alpha \beta} D^{\lambda}\right] \phi_{\lambda \beta \mu_{3} \cdots \mu_{s}}^{s}+m^{2} \epsilon^{\mu_{2} \cdots \mu_{s}} D^{\lambda} \phi_{\lambda \mu_{2} \cdots \mu_{s}}^{s}\right\}
\end{aligned}
$$

which confirms and extends the result found in [6].

Now, to eliminate the unwanted curvature derivative terms in (3.8), one needs to add truly nonminimal terms to the action (either the minimal one obtained with the order of derivatives specified above or the nonminimal one previously introduced, that is $S=S_{m}+S_{n m}$ ). After a straightforward but tedious calculation they turn out to be the sum $\Delta S=\Delta S_{1}+\Delta S_{2}+\Delta S_{3}$ of three contributions:

$$
\begin{aligned}
\Delta S_{1} & =\frac{s(s-1)(s-2)}{2 m^{2}} \int d^{4} x \sqrt{-g}\left[\partial^{\lambda} \phi_{\alpha \gamma \lambda}^{s}{ }^{\mu_{4} \cdots \mu_{s}}\left(\partial^{\rho} R^{\alpha \beta \gamma \delta}\right) \phi_{\beta \delta \rho \mu_{4} \cdots \mu_{s}}^{s}-\phi_{\alpha \gamma \lambda}^{s}{ }^{\mu_{4} \cdots \mu_{s}} R^{\alpha \beta \gamma \delta} \partial^{\lambda} \partial^{\rho} \phi_{\beta \delta \rho \mu_{4} \cdots \mu_{s}}^{s}\right], \\
\Delta S_{2} & =\frac{2 s(s-1)^{2}(s-2)}{m^{2}(2 s-1)} \int d^{4} x \sqrt{-g}\left[\phi_{\alpha}^{s-2 \mu_{2} \cdots \mu_{s-2}}\left(\partial^{\rho} R^{\alpha \beta \gamma \delta}\right) \partial_{\gamma} \phi_{\rho \mu_{2} \cdots \mu_{s-2} \beta \delta}^{s}\right] \\
\Delta S_{3} & =\frac{s(s-1)^{2}(s-2)(s-3)}{m^{2}(2 s-1)} \int d^{4} x \sqrt{-g}\left[\phi_{\alpha \gamma}^{s-2 \mu_{3} \cdots \mu_{s-2}}\left(\partial^{\lambda} \partial^{\rho} R^{\alpha \beta \gamma \delta}\right) \phi_{\beta \delta \mu_{3} \cdots \mu_{s-2} \lambda \rho}^{s}\right] .
\end{aligned}
$$

In this way we find the variation of the complete action $S_{T}=S+\Delta S$ to be 


$$
\delta S_{T}=\int d^{4} x \sqrt{-g} \frac{(s-1)(s-2)}{m^{2}} \epsilon_{\alpha \gamma}^{\mu_{3} \cdots \mu_{s-1}}\left(\partial^{2}-m^{2}\right)\left[\left(\partial^{\rho} R^{\alpha \beta \gamma \delta}\right) \phi_{\beta \delta \mu_{3} \cdots \mu_{s-1} \rho}^{s}\right]+\operatorname{soft} \operatorname{terms}+O\left(h^{2}\right)
$$

In this variation there is only one hard term (vanishing for spin $s<3$ ) and it is proportional to $\partial^{2}-m^{2}$. When inserted in the expression for the scattering amplitude $J \Pi J$, it gives rise to a local "seagull" term that can be canceled by adding local counterterms $O\left(h^{2}\right)$, and therefore it can be neglected. This is the new feature that appears at spin 3: the longitudinal part of the current $J$ need not be soft, but it may contain hard parts as long as they are proportional to the equations of motion of the field $\phi$; these only give rise to "seagull" diagrams that can be consistently ignored. Another way of stating this property is that terms proportional to the equations of motion of $\phi$ can be eliminated by a local field redefinition of $\phi$, which does not change the form of the free Lagrangian. Finally, notice that the $\Delta S$ of (3.11) vanish on the free $\phi$ shell, where $\partial \cdot \phi^{s}=0=\phi^{s-2}$. This means that they do not contribute to such physical quantities as the higher gravitational multipoles.

\section{HALF-INTEGER SPINS}

To study the fermionic systems, we follow the formulation of [8] (slightly different from that of Sec. II) and write the free flat-space Lagrangian for massive particles of half-integer spin $s=n+\frac{1}{2}$ as

$$
\begin{aligned}
L= & \bar{\psi}^{n}(\not \partial-m) \psi^{n}+\frac{2 n^{2}}{2 n+1}\left[\bar{\psi}^{n-1} \partial \cdot \psi^{n}-\bar{\psi}^{n} \cdot \partial \psi^{n-1}\right. \\
& \left.+\bar{\psi}^{n-1}\left(\not \partial+\frac{n+1}{n} m\right) \psi^{n-1}+\cdots\right]
\end{aligned}
$$

The fields $\psi^{p}$ are the minimal set needed to represent the physical degrees of freedom of a pure spin- $s$ free massive particle. They are symmetric $\gamma$-traceless tensor-spinors of rank $p=n, n-1, n-2, \ldots, 0$; for rank lower than $n-1$, they appear in $L$ with multiplicity 2 (namely $\psi^{i, p}$ and $i=$ $1,2)$. Here the metric is the Pauli metric $g^{\mu \nu}=\delta^{\mu \nu}$ and the convention on the $\gamma$ matrices (see [7]) is

$$
\gamma^{\mu} \gamma^{\nu}+\gamma^{\nu} \gamma^{\mu}=2 \delta^{\mu \nu}, \quad \gamma^{\mu^{\dagger}}=\gamma^{\mu}, \quad \gamma^{5}=\gamma^{1} \gamma^{2} \gamma^{3} \gamma^{4}
$$

Note that since $\psi^{p}$ is symmetric and $\gamma$-traceless, it is automatically also traceless: $\psi_{\mu \mu_{3} \cdots \mu_{p}}^{p \mu}=0$. The free equations of motion derived from (4.1) are

$$
\begin{gathered}
(\not \partial-m) \psi^{n}=0, \quad \partial \cdot \psi^{n}=0, \quad \psi^{n-1}=0=\psi^{i, p}, \\
p<n-1, \quad i=1,2 .
\end{gathered}
$$

The free massless Lagrangian possesses a restricted gauge transformation [3] given by

$$
\begin{aligned}
\delta \psi_{\mu_{1} \cdots \mu_{n}}^{n}= & \frac{2 n(n+1)}{2 n+1} \partial_{\left(\mu_{1}\right.} \epsilon_{\left.\mu_{2} \mu_{3} \cdots \mu_{n}\right)} \\
& -\frac{n}{2 n+1} \gamma_{\left(\mu_{1}\right.} \not \partial \epsilon_{\left.\mu_{2} \mu_{3} \cdots \mu_{n}\right)}, \\
\delta \psi_{\mu_{1} \cdots \mu_{n-1}}^{n-1}= & \not \partial \epsilon_{\mu_{1} \cdots \mu_{n-1}} \\
\delta \psi^{i, p}= & 0, \quad i=1,2, \text { and } \forall p<n-1,
\end{aligned}
$$

where $\epsilon$ is a rank- $(n-1)$ symmetric tensor spinor that is $\gamma$-traceless and divergenceless. While half-integer spins have unique minimal coupling to gravity, they share the tree unitarity problems of spin $\frac{5}{2}$ discussed in Sec. II. In fact, the variation of the minimal action $S_{m}$ under the transformation (4.4), linearized in the gravitational field and with the fields $\psi^{p}$ and $h$ put on free shell, gives

$$
\begin{aligned}
\delta S_{m} & =\int d^{4} x \sqrt{g} \frac{4 n\left(n^{2}-1\right)}{2 n+1} \bar{\epsilon}^{\alpha \mu_{3} \cdots \mu_{n}} \gamma^{\lambda} R_{\alpha \beta \lambda \delta} \psi_{\mu_{3} \cdots \mu_{n}}^{n \beta \delta}+\text { soft terms }+O\left(h^{2}\right) \\
& =\int d^{4} x \sqrt{g} \frac{4 n\left(n^{2}-1\right)}{2 n+1} \frac{1}{m} \bar{\epsilon}^{\alpha \mu_{3} \cdots \mu_{n}} R_{\alpha \beta \lambda \delta}^{+} \partial^{\lambda} \psi_{\mu_{3} \cdots \mu_{n}}^{n \beta \delta}+\text { soft terms }+O\left(h^{2}\right)
\end{aligned}
$$

By performing the same procedure as used in the previous section, we can remove the hard term in Eq. (4.5); here we add the nonminimal term

$$
S_{m n}=\frac{n(n-1)}{2 m} \int d^{4} x \sqrt{g} \bar{\psi}^{n \alpha \gamma \mu_{3} \cdots \mu_{n}} R_{\alpha \beta \gamma \delta}^{+} \psi_{\mu_{3} \cdots \mu_{n}}^{n \beta \delta}
$$

This result confirms [4]; in analogy with the bosonic case, for spin $s \geq \frac{7}{2}$ (i.e., $n \geq 3$ ), the variation of the new nonminimal action gives rise to additional hard terms. They read

$$
\delta S=-\frac{2 n\left(n^{2}-1\right)(n-2)}{m(2 n+1)} \int d^{4} x \sqrt{g} \bar{\epsilon}^{\alpha \gamma \mu_{3} \cdots \mu_{n-1}}\left(\partial^{\lambda} R_{\alpha \beta \gamma \delta}^{+}\right) \psi_{\mu_{3} \cdots \mu_{n-1} \lambda}^{n \beta \delta} .
$$

These terms can be canceled, as in the bosonic case, by introducing extra terms, which again vanish on shell (4.3): 


$$
\Delta S=\frac{2 n\left(n^{2}-1\right)(n-2)}{m^{3}(2 n+1)} \int d^{4} x \sqrt{g}\left\{\bar{\psi}^{n-1 \alpha \gamma \mu_{3} \cdots \mu_{n-1}}\left[\partial^{\lambda}(m+\not \partial) R_{\alpha \beta \gamma \delta}^{-}\right] \psi^{n \beta \delta}{ }_{\mu_{3} \cdots \mu_{n-1} \lambda}\right\} .
$$

The variation of the total action $S_{m}+S_{n m}+\Delta S$ now gives

$$
\delta S=\int d^{4} x \sqrt{g} \frac{2 n\left(n^{2}-1\right)(n-2)}{(2 n+1) m^{3}} \bar{\epsilon}^{\alpha \gamma \mu_{3} \cdots \mu_{n-1}}\left(\partial^{2}-m^{2}\right)\left[\left(\partial^{\lambda} R_{\alpha \beta \gamma \delta}^{+}\right) \psi_{\mu_{3} \cdots \mu_{n-1} \lambda}^{n \beta \delta}+\operatorname{soft} \text { terms }+O\left(h^{2}\right) .\right.
$$

The remarks made about integer spin at the end of the previous section apply here as well: the terms proportional to the equations of motion of $\psi$ are irrelevant since they give rise to local "seagull" diagrams, and the additional terms in the Lagrangian, given in (4.8), do not contribute to the gravitational multipoles of the spin-s particle since they vanish on shell and can be eliminated by a local redefinition of $\psi$, which changes neither the kinetic term nor the $S$ matrix.

\section{CONCLUSIONS}

We have established the following "low-energy" theorem: for all massive, higher-spin fields that one can adequately soften the behavior of their forward elastic scattering amplitudes $f_{-}\left(E, s_{3}\right)$ to maintain tree-level unitarity up to the Planck scale. This was carried out explicitly and uniformly through the addition of nonminimal terms proportional to the Riemann (or Weyl) tensor and its derivatives. Equivalently, this means that the projection in the gauge direction (of the massless limit) of the improved matter field equations obeyed Bianchi identities not involving explicit curvature terms. We noted, however, that the complementary, gauge-orthogonal, contributions to the vertices (as well as the propagators) now contain inverse powers of mass and hence the corresponding amplitudes will still violate unitarity at lower scales; but conjecture that improvements here (if they can be achieved at all) will require taking into account couplings of infinite towers of massive particles. We emphasize that the transverse and longitudinal currents give rise to different pathologies. In particular, since $f_{-}\left(E, s_{3}\right)$ does not depend on spins higher than $s$, it is only sensitive to the longitudinal current. Thus, a necessary condition for tree-level unitarity is that this current be improved so as to become softly broken. On the other hand the transverse-current pathologies could be removed, at least in principle, by adding new higher-spin massive states, an addition that cannot help the longitudinal current. The latter must be, and was, improved entirely within the single-field framework.

Two open questions are particularly interesting. First, how do our new terms compare with the way closed string theory organizes its higher-spin excitations, in the domain in which such an expansion is valid? Its beta function, expanded to include these massive modes, should show both the longitudinal compensation terms given here as well as the more complicated transverse corrections we conjectured. The second question involves the gravitational quadrupole interactions implied by our nonminimal terms. Is there a universal value, similar to $g=\mathbf{2}$ in the corresponding electromagnetic higher-spin analysis, of the total static gravitational quadrupole moments?

\section{ACKNOWLEDGMENTS}

S.D. was supported in part by the NSF under Grants No. PHY 93-15811 and No. PHY 92-45316 and by the Ambrose Monell Foundation, at the Institute for Advanced Study; M.P. was supported in part by NSF under Grant No. PHY 93-18781. We gratefully acknowledge the pioneering and fundamental contributions to this, as to so many other parts of physics, by the late Julian Schwinger.
[1] C. Aragone and S. Deser, Phys. Lett. 86B, 161 (1979).

[2] E. S. Fradkin and M. A. Vasilev, Phys. Lett. B 189, 89 (1987); M. A. Vasilev, ibid. 243, 378 (1990).

[3] S. Ferrara, M. Porrati, and V. L. Telegdi, Phys. Rev. D 46, 3529 (1992).

[4] M. Porrati, Phys. Lett. B 304, 77 (1993).

[5] S. Deser and B. Zumino, Phys. Lett. 62B, 335 (1976).

[6] C. Aragone and S. Deser, Nuovo Cimento B57, 33 (1980).

[7] P. van Nieuwenhuizen, Phys. Rep. 68, 189 (1981).

[8] L. P. S. Singh and C. R. Hagen, Phys. Rev. D 9, 898 (1974); 9, 910 (1974).

[9] F. A. Berends, J. W. van Holten, B. de Wit, and P. van Nieuwenhuizen, Phys. Lett. 83B, 188 (1979); Nucl. Phys. B154, 261 (1979); J. Phys. A 13, 1643 (1980).

[10] J. Schwinger, Particles, Sources, and Fields (AddisonWesley, Reading, MA, 1970), Vol. I.

[11] J. M. Cornwall, D. N. Levin, and G. Tiktopoulos, Phys.
Rev. D 10, 1145 (1974); 11, 972 (1975); J. M. Cornwall and G. Tiktopoulos, Phys. Rev. Lett. 35, 338 (1975).

[12] J. Scherk and J. H. Schwarz, Nucl. Phys. B81, 118 (1974).

[13] S. Weinberg, in Lectures on Elementary Particles and Quantum Field Theory, edited by S. Deser et al. (MIT Press, Cambridge, MA, 1970), p. 285.

[14] C. H. Llewellyn Smith, Phys. Lett. 46B, 233 (1973).

[15] D. J. Gross, Phys. Rev. Lett. 60, 1229 (1988).

[16] J. Isberg, U. Lindström, and B. Sundborg, Phys. Lett. B 293, 321 (1992); J. Isberg, U. Lindström, B. Sundborg, and G. Theodoridis, Nucl. Phys. B411, 122 (1994).

[17] J. J. Atick and E. Witten, Nucl. Phys. B310, 291 (1988); E. Witten, Philos. Trans. R. Soc. London, Ser. A 320, 349 (1989).

[18] C. Aragone and H. La Roche, Nuovo Cimento 72A, 149 (1982). 